\title{
Use of a Customized Device for Correction of Antebrachial Angular Deformity in a Dog
}

\author{
C Iván Serra ${ }^{1,2 *}$, Luis Doménech ${ }^{3}$, Julio Soriano ${ }^{3}$, Aida Fages ${ }^{2}$, Víctor Moratalla ${ }^{4}$ and Carme Soler ${ }^{1,2}$ \\ ${ }^{1}$ Departamento Medicina y Cirugía Animal, Catholic University of Valencia, Spain \\ ${ }^{2}$ Hospital Veterinario Universitario, Catholic University of Valencia, Spain \\ ${ }^{3}$ Building Engineering and Industrial Production Department, Univerity CEU Cardenal Herrera, Spain \\ ${ }^{4}$ Orthopedic Service, Spain
}

*Corresponding author: C Iván Serra Aguado, Department of Animal Medicine and Surgery, Universidad Católica de Valencia, Avda Pérez Galdós, 51. 46018 Valencia, Spain

To Cite This Article: CI Serra, Luis D, Julio S, Aida F, Víctor Moratalla, et al., Use of a Customized Device for Correction of Antebrachial Angular Deformity in a Dog. 2020 - 8(3). AJBSR.MS.ID.001270. DOI: 10.34297/AJBSR.2020.08.001270.

Received: 誹 March 10, 2020; Published: 眥 April 03, 2020

\begin{abstract}
Angular deformity is a common condition in dogs that can cause serious malformations and is a challenge for surgeons to correct. A 15-monthold male Saint Bernard was evaluated due toright foreleg lameness. Orthopedic examination revealed a valgus deformity with external rotation and anteversion of the forelimb. Carpal examination revealedthe decreased range of motion with slight disturbances in carpal flexion. Radiography of both forelimbs showed angular deviation because of possible premature physeal closure of the distal ulnar growth plate.Surgical intervention was selected to correct the angular deformity. A closed-wedge osteotomy was planned using 3D reconstruction, obtained via computed tomography (CT). In order to accomplish this, a custom-made device was developed to aid the surgeon in establishing the position and orientation of the cutting planes during the intervention.Long-term follow-up obtained 3 years after surgery revealed the correct ossification of the osteotomy and complete resolution of lameness. The main advantages of using a customized device obtained from 3D CT include the predictability and accuracy of the method. The device allows for correction of atriple-axis angular long bone deviation, with full opposition between the bone fragments after osteotomy and minor loss of length of the limb.
\end{abstract}

Keywords: Angular, Deformity, Customized device, Computed tomography, Surgery, Dog

Abbreviations: CT: Computed Tomography; ABS: Acrylomitrile Butadiene Styrene; CORA: Center of Rotation of Angulation.

\section{Introduction}

Angular deformities of long bones represent a significant challenge for surgeons in both veterinary and human medicine. The origins of these deformities may be alterations in the metaphysis of young animals or mal-unions as a result of old fractures [1].

In many circumstances, such deformities can induce short-, medium-, and long-term functional and aesthetic alterations. The severity of these changes determines the need for surgical therapy. In general, surgery is indicated in cases where altered mobility and exercise intolerance are detected, or in order to reduce the progression of osteoarthritis [2].

Different surgical techniques for correcting angular limb deformation have been described such as external skeletal fixators, dome osteotomies, or open and closed wedge [1-5]. Traditionally these techniques are planned from radiologic studies using orthogonal radiographs. However, the use of a CT has allowed 3-D images to be obtained that provide advanced information about the patient, especially complex geometries. In addition, CT has demonstrated advantages in the diagnosis of angular deformities [6-9].

One of the main challenges for a surgeon doing this type of operation is executing the surgical plan as accurately as possible $[1,2]$. The execution is based on the use of anatomical reference points, which are not easily accessible. In this circumstance, the use of a custom device, developed using the full knowledge of the patient that is provided via CT, enables the surgeon to accurately perform the necessary correction. 


\section{Clinical Report}

A 15-month-old, $85 \mathrm{~kg}$ male Saint Bernard presented with a chronic history of right forelimb lameness due to a valgus deformity, which had worsened in the preceding 3 months. On physical examination, vital parameters were within normal limits. The orthopedic examination revealed right valgus deformity with external rotation and ante-version of the forearm. Examination of the carpus revealed the decreased range of motion with slight disturbances in carpal flexion. Examination of the elbow revealed no obvious abnormality.

Radiography of both forelimbs (radius and ulna including the proximal and distal joint) revealed right forelimb angular deviation because of a possible premature physeal closure of the distal ulnar growth plate (Figure 1A).

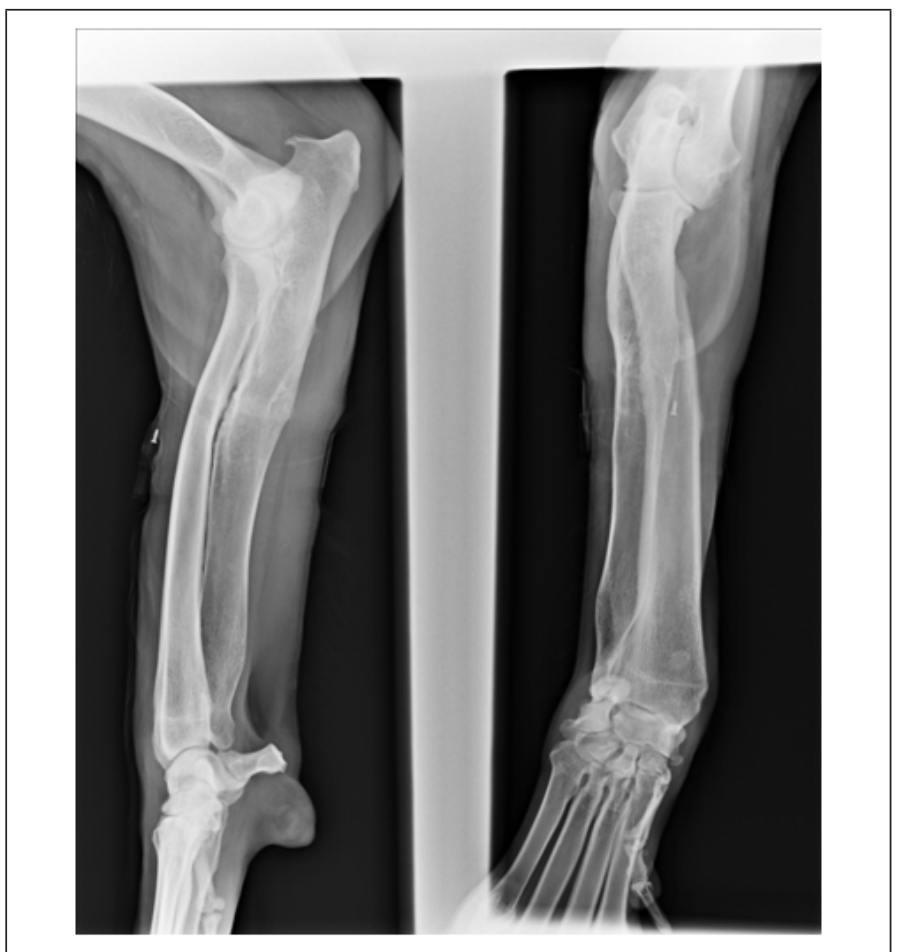

Figure 1A: Surgical planning: cranio-caudal and medio-lateral radiographs of right antebrachium at the preoperative moment.

Surgical correction was prescribed; the preoperative planning consisted of developing a customized device to aid the surgeon during surgery. A CT study of both forelimbs was carried out, including the elbow and carpal joints. The dog was placed in sternal recumbency with the forelimbs extended cranially, allowing the carpi and metacarpi toremain in a neutral position. A CT scan was performed in a 2-slice CT scanner witha $1 \mathrm{~mm}$ gap between cuts, starting in the proximal humerus, and extending to include the metacarpi. The CT findings wereexported to DICOM format, allowing further manipulation, resulting in a 3D reconstruction of the pathological limb (Figure 1B).

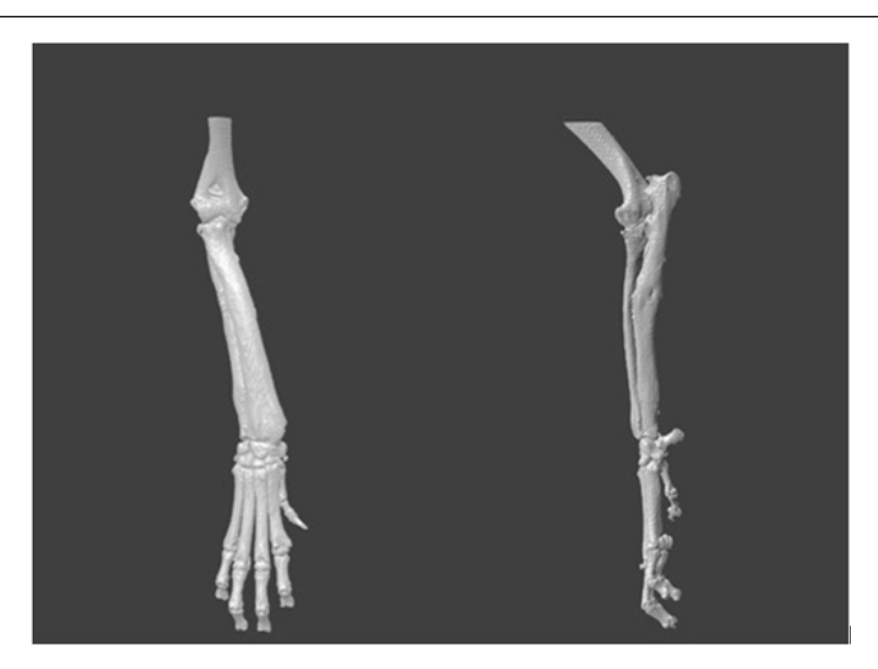

Figure 1B: Three-dimensional CT reconstruction of the right Antebrachium.

The method used to plan the surgery is described by Domenech et al. [10]. This methodallows for correction of angular deformity in a long bone by means ofclosed wedge ostectomies. Thismethod aims to minimize loss of bone length. This methodology only requires the availability of 3-D reconstruction of the diseased bone and the correct definition of the proximal and distal joint planes (Figure 1C). In this case, the 3-D model was obtained by reconstruction information obtained by CT, which was then examined by a surgeon. A surgical plan was formed based on the bone's anatomical points, which was determined the joint planes.

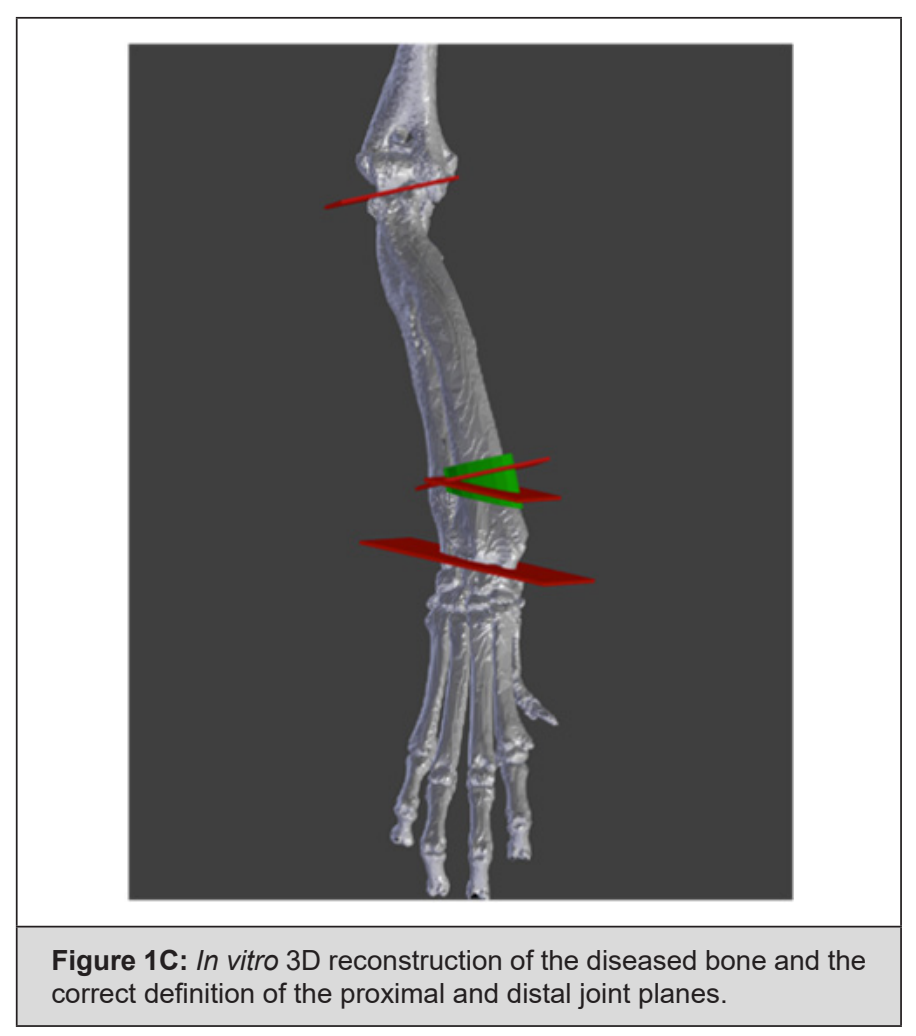


The device was designed to help the surgeon accurately perform the planned operation (Figure 1D). Therefore, the main requirement of the device was the accurate definition of all of the surgical actions, such as cutting planes and/or rotations. In addition, since the device interacts with the patient's body, the design had to be movable among tissues and minimally invasive. To this effect, the device could not significantly expand the boarding area, have sharp points, or be excessively thick. Given these parameters, the following requirements were established:

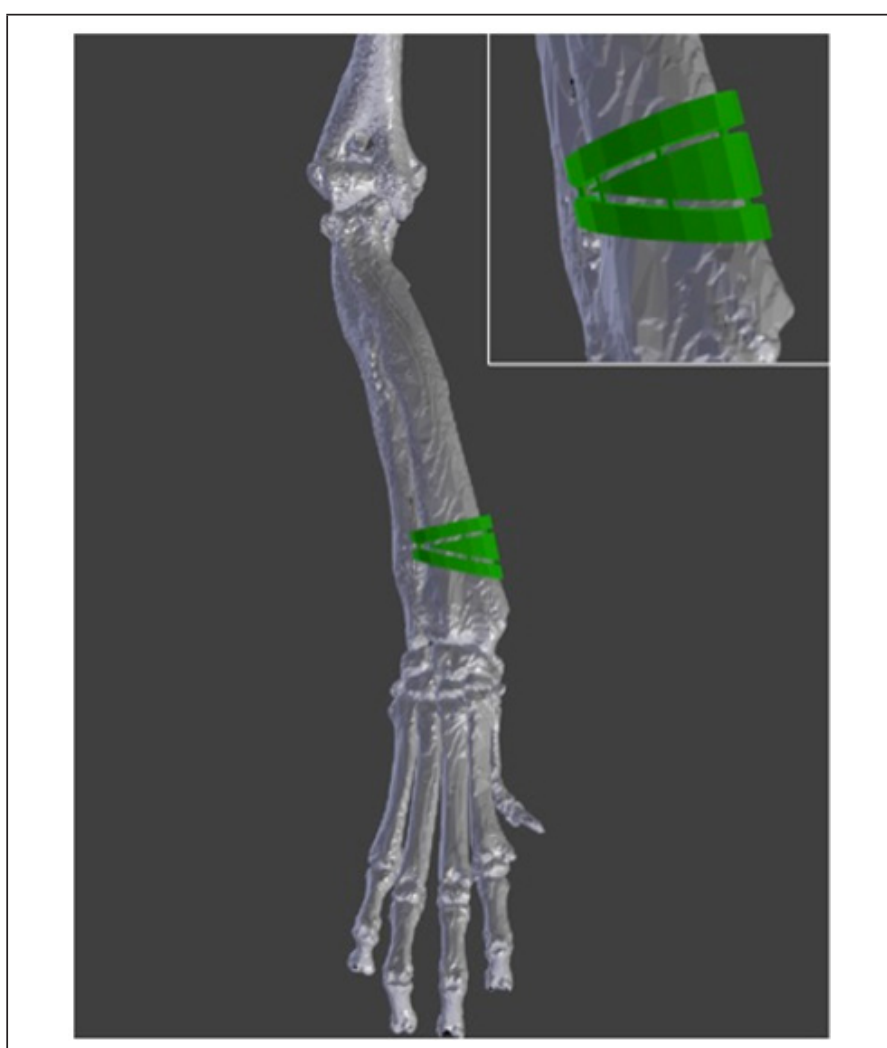

Figure 1D: The device design.

a. The device must be positioned with ease and accuracy.

b. The device must precisely indicate the orientation in which to make incisions.

c. The device must incorporate a system indicating the rotation to be applied when joining the fragments.

d. The device should be light and blunt.

e. The device must be minimally invasive.

f. The device must be autoclave proof.

Under these conditions, a custom-made, single-use guide device was designed (Figure 1D \& E). The internal aspect of the device was made using the bone's negative shape, with a snap-fit joint geometry to give an accurate location. The device was manufactured using rapid professional prototyping, with acrylonitrile butadiene styrene (ABS) material.

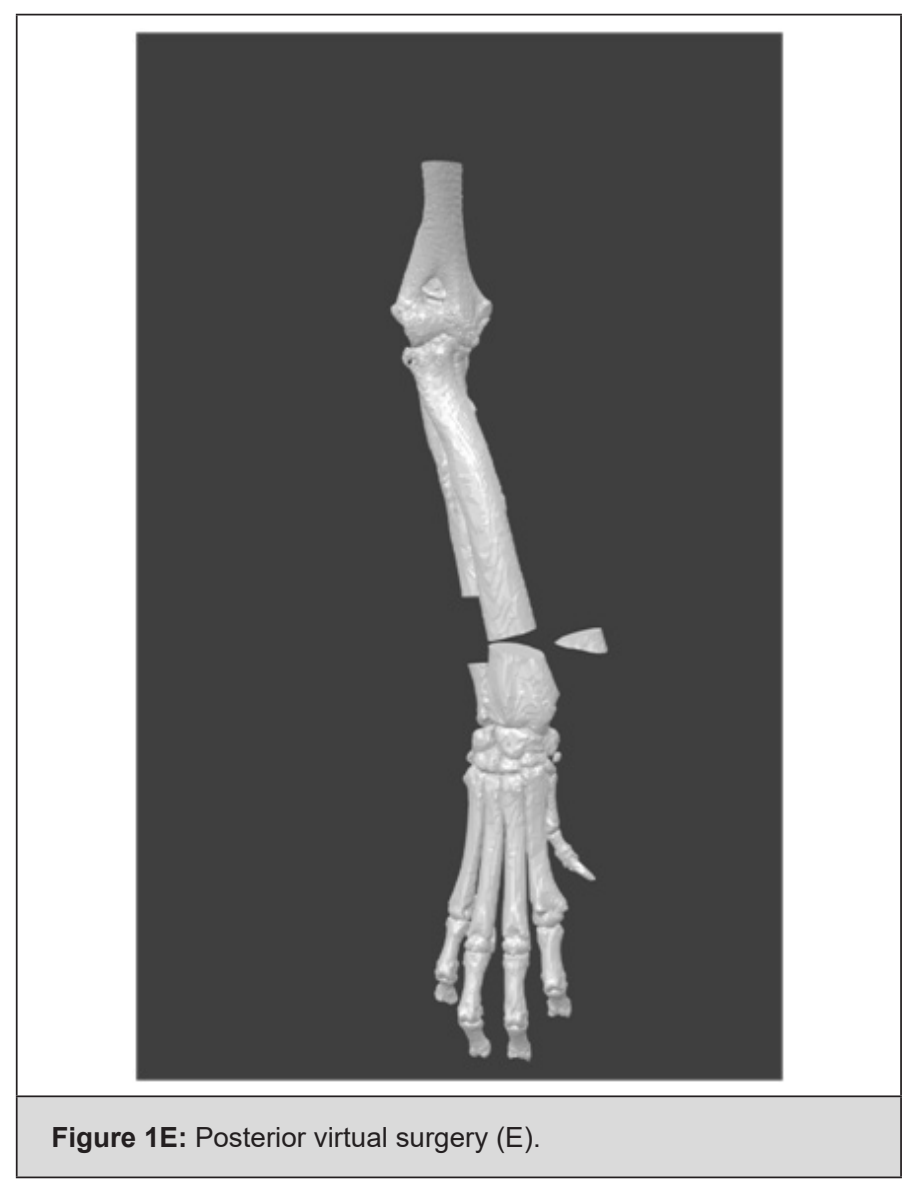

The next step consisted of testing the surgical device "in vitro." In order to achieve this, the guide device and affected bone 3D reconstruction were manufactured (Figure 1D). This allowedfor practice of the stabilization technique, which consisted of applying two locking plates for $3.5 \mathrm{~mm}$ diameter screws in the cranial aspect of the radius. Finally, after confirming the "in vitro" results, the clinical surgery was performed.

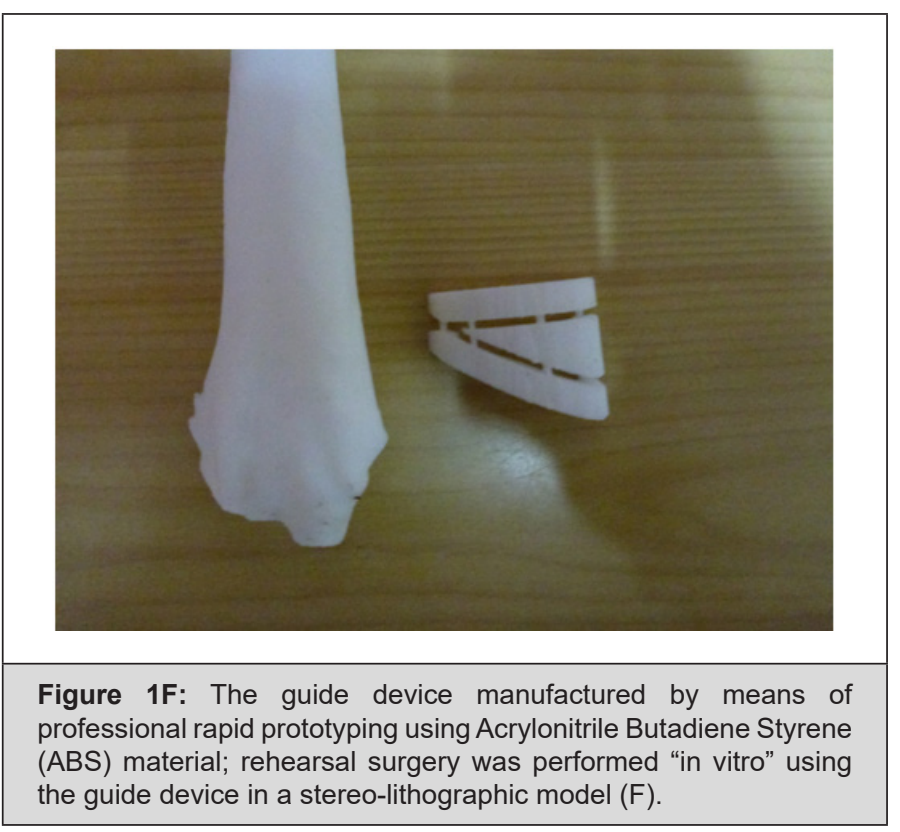


The anesthetic protocol consisted of premedication with dexmedetomidine $(0.005 \mathrm{mg} / \mathrm{kg}$, [IV]), combined with methadone $(0.2 \mathrm{mg} / \mathrm{kg}$ [IM]). Induction was performed with propofol $(3 \mathrm{mg} / \mathrm{kg}$ [IV]), andanesthesia was maintained with $1.5 \%$ isoflurane. The dog received a loading dose of fentanyl $(0.002 \mathrm{mg} / \mathrm{kg}$ [IV]), followed by constant rate infusion of $(0.005 \mathrm{mg} / \mathrm{kg} /$ hour [IV]), throughout the surgical procedure. Respiration was maintained using intermittent positive pressure ventilation with a mechanical ventilator.

The surgery was carried out through a lateral approach to the distal ulna to release the radius and allow the correct application of the device. A $3 \mathrm{~cm}$ ostectomy of the distal ulna was performed using an oscillating saw. Then, the opposition of the flexor and extensor muscles, subcutaneous tissue, and skin were closed.

Next, a cranio medial approach to the distal radius was performed to allow the preparation of the bone for placing the device and to accomplish the ostectomy and subsequent stabilization. Once the distal shaft of the radius was exposed, the device was set in place using a press-fit mechanism (Figure 2A) and fixed to the bone with two bone forceps. The ostectomy was performed using an oscillating saw, following the cutting planes established by the guide (Figure 2B). Once the bone fragments were reduced to their preset position, 10 - and 14-hole x $3.5 \mathrm{~mm}$ locking plates were contoured and fixed to the cranial aspect of the radius. After the stabilization was complete, routine closure was performed.

Figure 2A: Surgical intervention; during surgery, the guide device was held in the predetermined point of the radius by mean of a press-fit system.
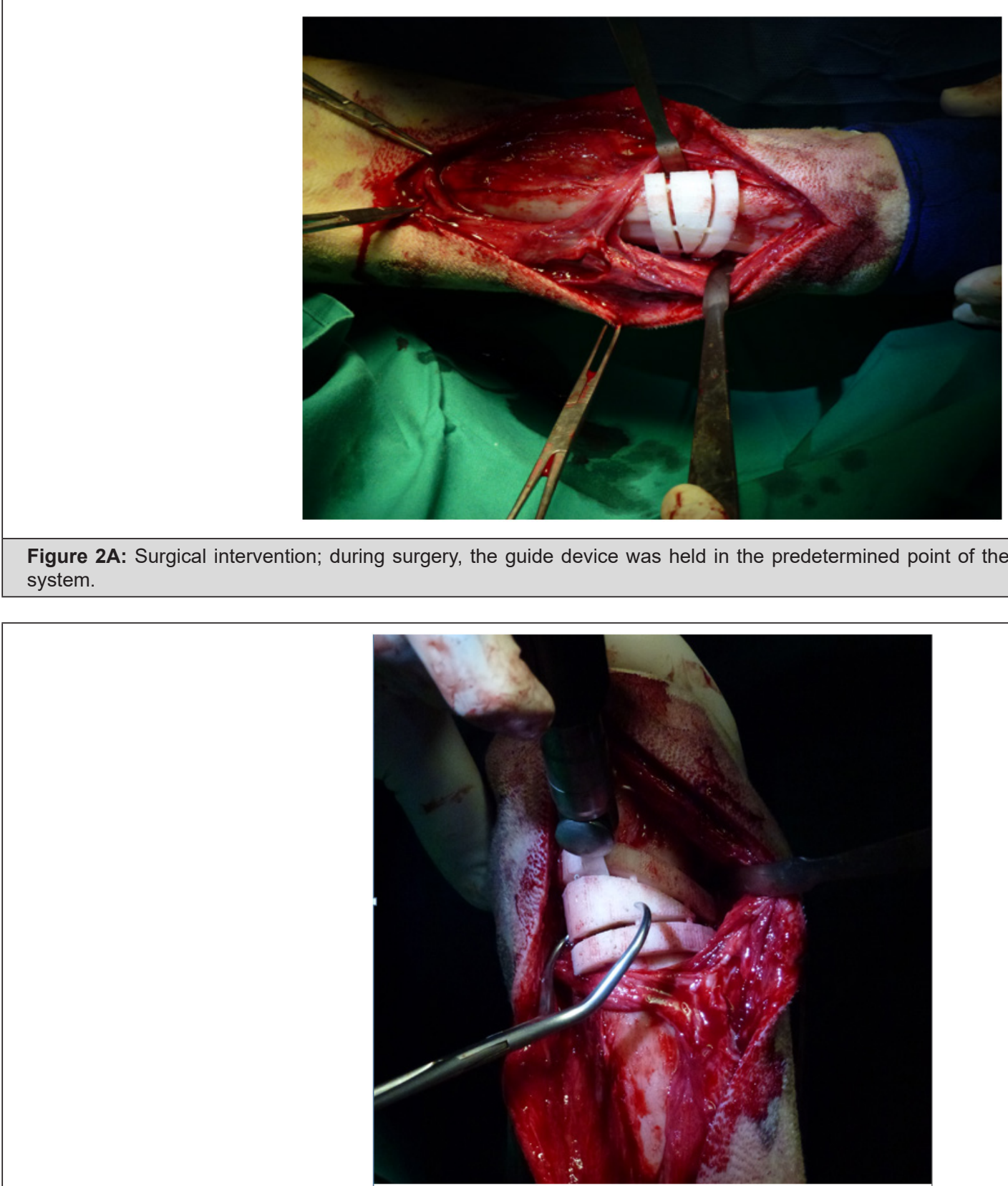

Figure 2B: Oscillating saw was used to cut the bone using the guide device (B), and finally both fragments were reduced and stabilized as in the "in vitro" surgery. 
Postsurgical treatment consisted of cephalexin $20 \mathrm{mg} / \mathrm{kg}$ (PO) q $12 \mathrm{hr}$ for 10 days, omeprazole $0.7 \mathrm{mg} / \mathrm{kg}$ (PO) q $24 \mathrm{hr}$ for 21 days, and robenacoxib $2 \mathrm{mg} / \mathrm{kg}$ (PO)q $24 \mathrm{hr}$ for 21 days. Methadone 0.2 $\mathrm{mg} / \mathrm{kg}(\mathrm{IM})$ was prescribed $\mathrm{q} 4 \mathrm{hr}$ during the first $24 \mathrm{hr}$, followed by buprenorphine $0,006 \mathrm{mg} / \mathrm{kg}(\mathrm{PO}) \mathrm{q}$ 8hr for 5 days after withdrawing the methadone. A Robert-Jones bandage was applied for the first two postoperative weeks.

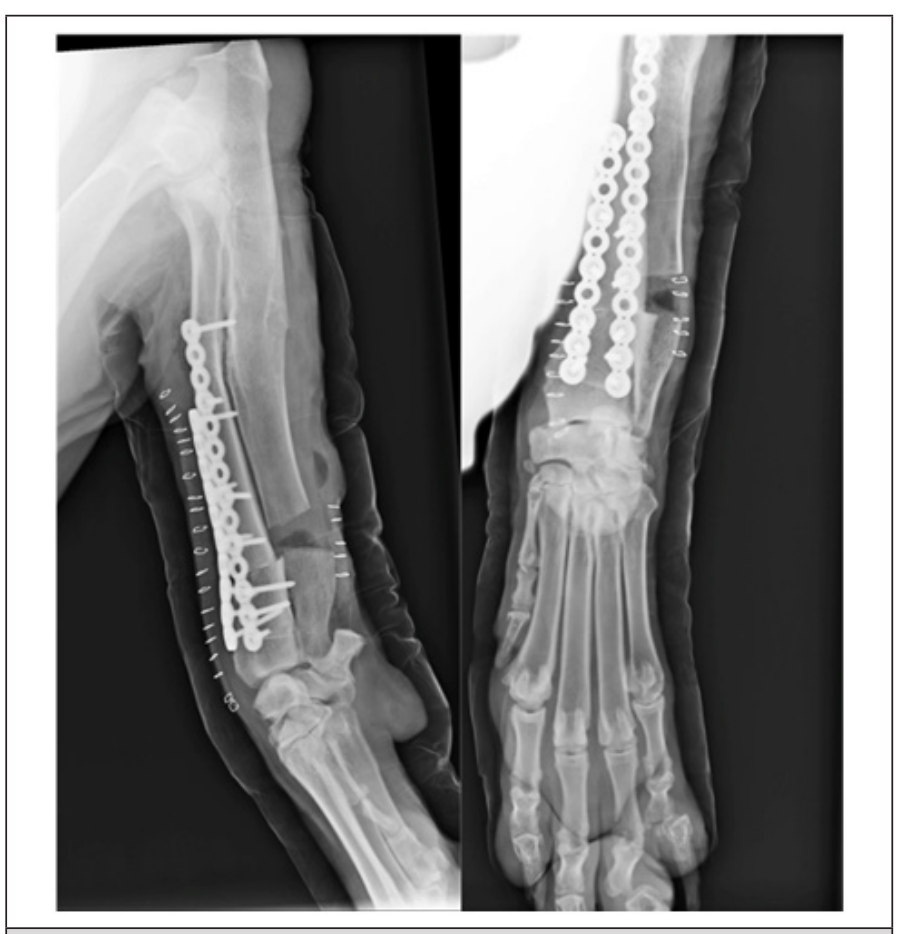

Figure 2C: Postoperative radiological appearance.

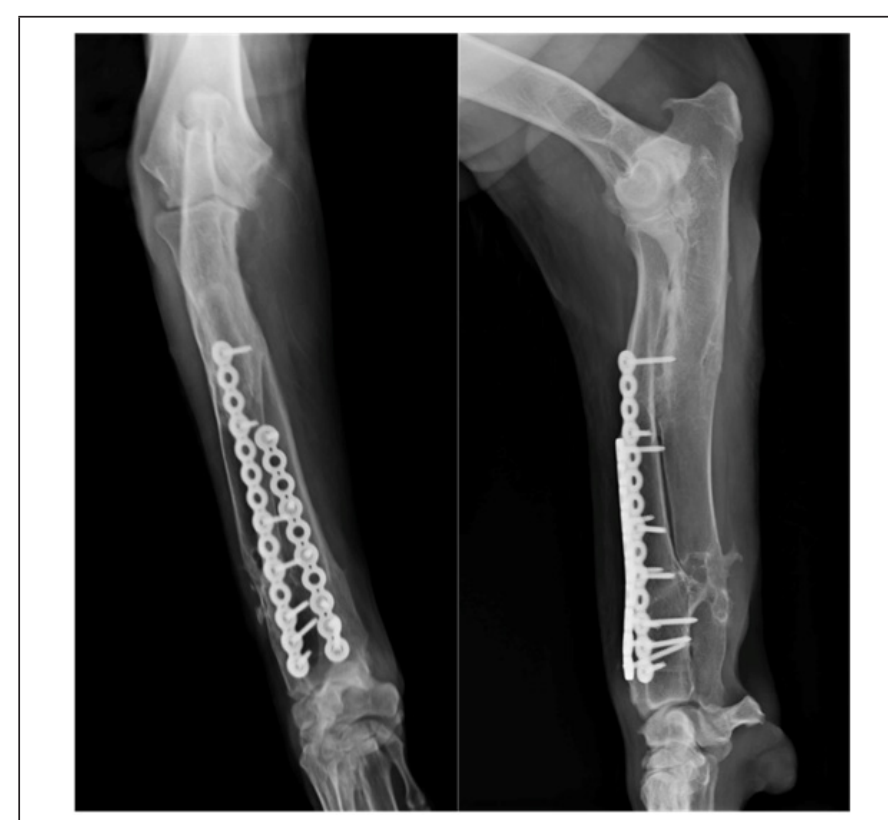

Figure 2D: Follow-up radiographs 3 years after surgery (D).

Follow-up radiographs (Figure 2C) were performed at 4, 8, 12,16 and 24 weeks post-surgery, at which point no anomalies in bone healing were detected. Orthopedic examination revealed immediate correct alignment and normal weight bearing of the limb, with the resolution of lameness 16 weeks post-operatively. Three years postoperatively, an orthopedic exam and radiography were performed (Figure 2D). This revealed the satisfactory resolution of clinical signs with no lameness. At this time, elbow range of motion was normal. A minor reduction in carpal range of motion was detected but was non-painful.

\section{Discussion}

We describe the execution of a closing wedge ostectomy. This procedure is based on 3-D planning and a custom-designed device for guiding the surgeon during the operation. Rapid prototyping was used to manufacture the device and for the "in vitro" test.

The surgical intervention to correct long bone angular deformities remains a challenge for surgeons. The main difficulty is the practical translation of the designed corrective measurements into an intra-operative incision [11]. This gap in knowledge, along with the complexity of the surgery itself, increases the probability of human error.

A diverse range of techniques can be applied to correct angular deformities, such as linear osteotomies, dome osteotomies, wedge ostectomies, and stapling $[5,11,12]$. Several stabilization methods are available, such as external and internal fixation systems $[13,14]$. The center of rotation of angulation (CORA) calculation is the most widely used method for planning surgical interventions [15].

In this paper, the authors describe the execution of a closing wedge ostectomy. The planning was developed using the strategy described by Domenech et al. [10]. This method was selected due to its ability to minimize bone loss, which is an intrinsic characteristic of closed wedge ostectomy. A guiding device was developed using the position and cutting planes provided by this strategy. The main advantages of the deviceare that it can only beappliedto the correct region of the bone surface andit clearly designates the cutting planes, as well as the rotation to be applied.

The 3-D data obtained from the CT provided betterknowledge of the angular deformity. Moreover, the 3-D data allow forsimulation of the surgery via software and the creation of a manufactured copy that permits the execution of an "in vitro" intervention. The use of computer simulation has been reported in human and veterinary surgery [11-14]. Computer simulation makes it possible for the surgeon to experiment with cutting plane options and estimate implant positions.

The "in vitro" intervention also provided several advantages. It not only validated the accuracy of the simulated surgery but also exhibited other issues that were not detected in the simulated surgery. Issues raised included the compatibility between the guide and the bone and the compatibility between the surgeon's tools and the device [1-13]. 
The compatibility between the device and the bone was achieved by the manufacturing accuracy and the device geometry. The accuracy provided by the 3-D reconstruction and the rapid prototyping machine proved sufficient to reproduce the surface of the bone. The geometry of the guide wassufficiently concave to get an accurate fit and sufficiently convex to allow for correct placement.

The compatibility between the surgeon's tools and the device was accomplished by means of the manufacturing accuracy, the device geometry, and the manufacturing material. Even though it is single-use, the device should tolerate the vibrations of the saw, contain the holes and guides to aid the surgeon, and should not damage surgical instruments. The selected machinery and material proved sufficient for these requirements.

The described procedure was more accurate in terms of the surgical placement and the cutting planes. It also reduces the surgical time by avoiding the necessary intra-operative measurements. Moreover, the knowledge obtained during planning allows for pre-selection of all implants used in the stabilization stage. These characteristics improve the probability of surgical success [2].

Among the limitations of the method described are that the correction proposed is permanent and is therefore unsuitable for growing patients. Furthermore, the device needs a clean bone surface; thusbones with a thick fibro cartilaginous layer could affect mechanical compatibility, and malunion could be an example of this incompatibility.

\section{Conclusion}

The clinical use of a 3-D device has proven valid for correction of antebrachial angular deformity such as the one in the presented case. The clinical utility of a simulated and an "in vitro" intervention has been observed, and the results have been applied to an "in vivo" intervention with excellent results both short- and long-term. However, further studies with a larger sample size and other bone sites are required in order to develop wider conclusions.

\section{Acknowledgments}

The authors thank Let Pub for review of the manuscript. The authors did not receive any funding for this research or publication.

\section{Conflict of Interest}

Not declared.

\section{References}

1. Deruddere KJ, Snelling SR (2014) A retrospective review of antebrachial angular and rotational limb deformity correction in dogs using intraoperative alignment and type $1 \mathrm{~b}$ external fixation. N Z Vet J 62(5): 290-296.

2. Crosse KR, Worth AJ (2010) Computer-assisted surgical correction of an antebrachial deformity in a dog. Vet Comp Orthop Traumatol 5: 354-361.

3. Petazzoni M, Piras A, Jaeger GH, Marioni C (2009) Correction of rotational deformity of the pes with external skeletal fixation in four dogs. Vet Surg 38(4): 506-514.

4. Captug O, Bilgili H (2009) Correction of the antebrachial deformity using circular external skeletal fixator in 7 dogs. Pol J Vet Sci 12(1): 45-54.

5. Fox DB, Tomlinson JL, Cook JL, Breshears LM (2006) Principles of uniapical and biapical radial deformity correction using dome osteotomies and the center of rotation of angulation methodology in dogs. Vet Surg 35(1): 67-77.

6. Dismukes DI, Fox DB, Tomlinson JL, Essman SC (2008) Use of radiographic measures and three- dimensional computed tomographic imaging in surgical correction of an antebrachial deformity in a dog. J Am Vet Med Assoc 232(1): 68-73.

7. Leong NL, Buijze GA, Fu EC, Stockmans F, Jupiter JB (2010) Distal Radius Malunion (DiRaM) collaborative group. Computer-assisted versus noncomputer-assisted preoperative planning of corrective osteotomy for extra-articular distal radius malunions: a randomized controlled trial. BMC Musculoskelet Disord 11: 282.

8. Apelt D, Kowaleski MP, Dyce J (2005) Comparison of computed tomographic and standard radiographic determination of tibial torsion in the dog. Vet Surg 34(5): 457-462.

9. Cooley K, Kroner K, Muir P, Hetzel SJ, Bleedorn JA (2018) Assessment of overall thoracic limb axial alignment in dogs with antebrachial deformity. Vet Surg pp. 1-6.

10. Domenech L, Muñoz-Almaraz FJ, Serra CI, C Soler, N Montes (2016) A 3D mathematical model for planning ostectomy on long-bone angular deformities. Journal of Computational and Applied Mathematics 291: 58-65.

11. Smouha EE, Chen D, Li B, Liang Z (2001) Computer-aided virtual surgery for congenital aural atresia. Otol Neurotol 22(2): 178-182.

12. Dobbe JG, Pré KJ, Kloen P, Blankevoort L, Streekstra GJ (2011) Computerassisted and patient-specific 3-d planning and evaluation of a single-cut rotational osteotomy for complex long-bone deformities. Med Biol Eng Comput 4(12): 1363-1370.

13. Meyer DC, Siebenrock KA, Schiele B, Gerber C (2005) A new methodology for the planning of single-cut corrective osteotomies of mal-aligned long bones. Clin Biomech 20(2): 223-227.

14. Hudson CC, Lewis DD, Cross AR, Dunbar NJ, Horodyski M, et al. (2012) A biomechanical comparison of three hybrid linear-circular external fixator constructs. Vet Surg 41(8): 954-965.

15. Paley D, Herzenberg JE, McKie J, Bhave A. Tetsworth K, et al. (1994) Deformity planning for frontal and sagittal plane corrective osteotomies. Orthop Clin North Am 25(3): 425-465. 